\title{
31
}

\section{Educational resource management with a human interface}

\author{
Tuulikki Paturi \\ Institute of Information Technology, Rautatieläisenkatu 5, FIN-00520 Helsinki, \\ Finland
}

\begin{abstract}
In this paper, one example of designing schedules with a software package is descriped. The software is used to manage all the resources involved in scheduling. This application differs from other similar packages in that it assists the human mind in drawing up the best possible timetables rather than using any algorithm to automatically schedule the lessons.
\end{abstract}

Keyword Codes: J.1

Keywords: Adminstrative Data Processing

\section{BACKGROUND}

The Helsinki Business Polytechnic represents a new type of educational institution in Finland. The Polytechnic was founded and authorized by the Finnish Government in 1991. The institution amalgamates three colleges: The Finnish Business College, The Helsinki Secreterial College and The Institute of Information Technology (ATK-instituutti).

The Institute of Information Technology (IIT) was founded in 1970. It is a college which specializes in information technology and is owned and sponsored by the IIT Support Association. The Institute's main objective is to advance and develop the practical use of information technology by offering basic, advanced, and supplementary study programs. The Institute is also concerned with the development of education and teaching methods and is involved in research work in the fields of systems development and in the use of computers as tools in various work areas.

Today the IIT offers four different degree programs:

-The Degree Program in Information Technology consists of 140 credit units and covers a period of 3.5 years.

- The Datanomi Diploma Examination for adults in part-time attendance consists of 80 credit units and covers for a period of four years while students work in full-time employment.

- The 20 credit unit postgraduate program for DP professionals.

- The 20 credit unit postgraduate program for business college graduates.

Scheduling a term is a matter of allocating

- four degree program which will include 17 study groups with 6,000 contact lessons;

- 500 students;

- 35 full-time teachers and some 35 part-time teachers, and

- 13 class-rooms, 7 computer laboratories, 11 project rooms and 2 auditoria.

\section{SCHEDULING - THE PROCESS}

The curricula of our four degree programs are divided into teaching terms. Each term is 20 weeks long and is planned as a total unit. 


\subsection{The question of courses}

The scheduling begins with students planning their individual study programs for the coming term. They choose both the compulsory and optional courses from a "basket" of courses by filling in a form. The student office counts the number of participants in each course and delivers the information to the management.

The management decides which courses to give according to the level of interest shown by the students. At this point we know the number of lessons for the coming 1994 autumn term: they will amount to 6000 tutor-student contact hours.

\subsection{Staff numbers}

The teachers plan their individual teaching load for the following term. This may be reduced for individual research and development work in their own field of teaching. The load may therefore vary from term to term.

The teachers in Information Technology usually teach at most 304 lessons per term (an average of 16 lessons a week of which 8 are contact hours). If they participate in development projects the number of lessons can be anything between 0-304. Teachers in General Studies, Business and Economics usually teach from 361 hours ( 19 hours per week) up to 450 lessons a term. On average, we need 33 teachers for 12000 lessons; but in practice there are 35 fulltime teachers and over 30 part-time teachers.

\subsection{Preparing timetables}

The students register for both compulsory and free-choice courses. This is done by means of a school administration software program called Primus made by a Finnish company called StarSoft Ky. Primus keeps a record of the information concerning individual students' names and addresses, course selections and credits. It also includes all the information about the curricula, the subjects and their credit units. All this information is transferred to StarSoft Kurre which is used to design the timetables.

\subsection{Planning the study groups}

The students are divided into study groups (classes) according to their choices. This is done with Kurre, which assists in finding all the students that have the same combination of selections. They can then be formed into a study group or, if there are too many students, divide them into smaller groups which have all made the same selections. This procedure is iterative and is continued until a satisfactory result is reached. The idea is to create groups that are as independent as possible.

In figure 1 we see the grouping screen. There is a column for each student and a row for each subject. A white square means that the students have chosen the subject in question. Students are grouped by moving them from one class to another or by linking two or more classes together in one subject. Within a class the goal is to obtain white areas that are large as possible. In figure 1 the cursor is pointing at the third row, which shows three linked subjects: FYA/SAC/SAD. More details of the subjects are given on the lower right of the screen. There are 7/13/19 students studying those subjects and they are currently in classes 3a and $3 \mathrm{~b}$. Each subject has five lessons a week and these can be taught at the same time because only one student is attending any one of the linked subjects. The detail screen also shows the teachers and class-rooms accordingly. In this example the second subject has as yet neither teacher nor room. 


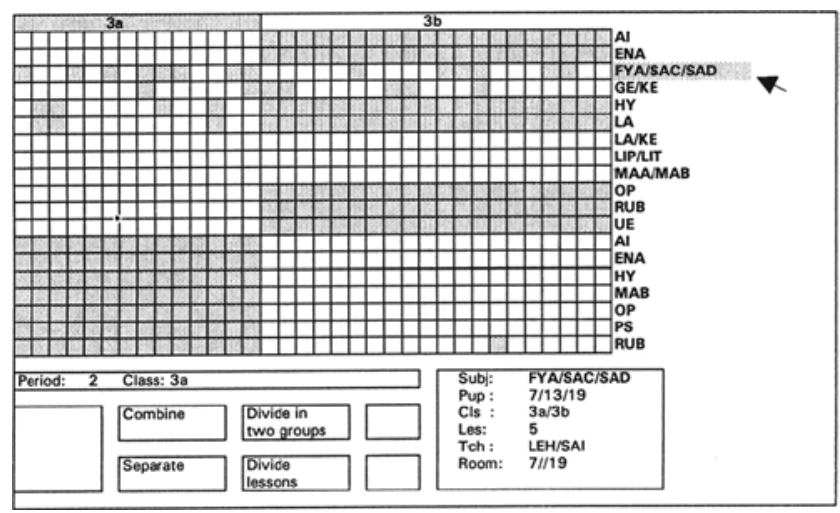

Figure 1. Grouping students according to their selections.

\subsection{Planning the periods}

The term of the Degree Programme in IT is divided into two periods. In this phase subjects are placed within the required period. It is also possible to change a subject from one period to another, thus, for instance, providing more evenly spread schedules for teachers.

\subsection{Planning the timetable}

Then comes the most challenging work: To place every 2-8 lesson block of four different degree programs on one week's timetable and to optimize the situation from any point of view. The primary goal is to give students a chance to study what they choose, to avoid empty hours in the middle of the students' day, to optimize the use of computer laboratories and other classrooms, to fullfil most of the teachers' wishes in planning the timetable. Everyone who is in charge of making timetables knows that these demands need small or sometimes even big miracles to come true. These miracles are performed with StarSoft Kurre. It does not automatically schedule the lessons according to any algorithm. Its main purpose is to support the human mind in making good timetables by keeping a record of the situation and preventing errors.

Arranging class schedules with StarSoft Kurre is easy and intuitive. The program instantly checks all the possible slots for a lesson and shows them clearly with a special color. Even if the lesson in question affects several classes and teachers, the screen at the time of placement shows miniature schedules for all parallel classes and for teachers involved with the lesson. Whenever the need arises, you can look at your school's overall situation in miniature on the screen. Every small detail can be enlarged to full size for closer inspection. The situtation can also be examined from three possible perspectives: the study group's, the classroom's and that of teacher availability. The decision to place a lesson is always made by the user. Human factors, too, can be taken into account in drawing up the best possible timetable.

Figure 2 shows a screen of arrangement. The problem at hand is to locate an object of three subjects for two classes and three teachers. The restrictions are that not all the teachers are available, students are occupied with some other subject, or, there is no class-room vacant. These restrictions are marked red (lighter gray in the figure 2). The program allows the user to put this object on any of the white rectangular slots. In the bottom half of the screen, a miniature schedule of the other class is shown which includes the three teachers and three classrooms involved. The miniature schedule of the fourth teacher (LEH) is chosen by the user. 
In the bottom left corner there are lists of classes, teachers and rooms. Any of them can be selected to obtain a miniature update of the current schedule in order to gain a wider overview.

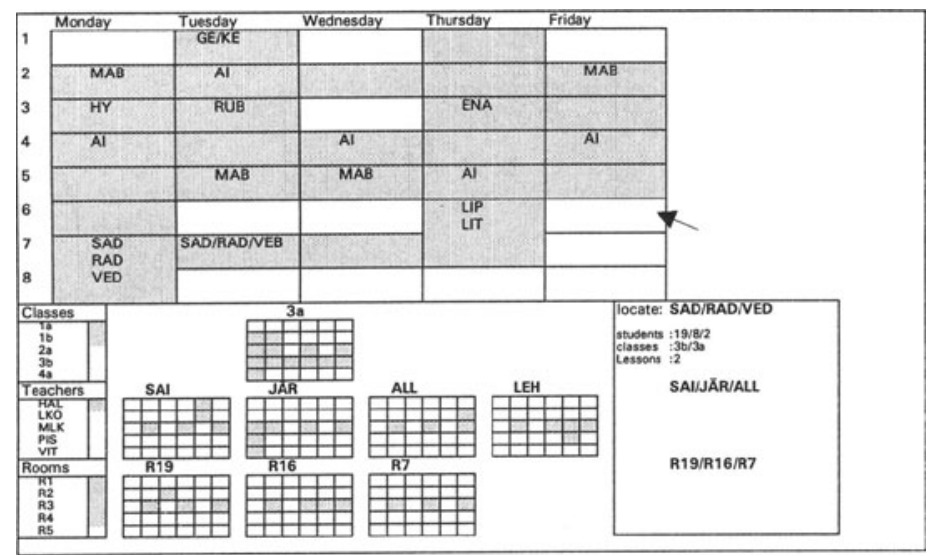

Figure 2. Arrangement of the lessons.

The program also has an autochoice function which can choose at any moment the most difficult lesson left to place in the schedule. Used frequently enough, autochoice ensures that lessons which are difficult to place are taken care of early on, thus reducing the chances of getting into a dead end situation.

In practice, I first mark so-called 'reserved times': Teachers may have commitments that prevent them from giving lessons at a certain time of the day or week. On the other hand, some part-time teachers and experts want to give their lessons at a certain time. I locate these lessons first.

The lessons can be placed by class, by teacher or by room. I usually start locating with the computer laboratories because they are the most wanted. After that I place teachers with greater time restrictions and then I place the lessons class by class. All through this the program supports me by showing the situation from any point of view and helps me to plan high quality schedules.

In the middle of arranging, the editor function in the arrangement program allows to change the input data, change subject labels and teacher and room designations, enter new information or remove old information, and so forth. All the necessary changes can be made as soon as the need to change appears.

\subsection{Printing the timetables}

The printing functions are very flexible. Already at the input stage data can be printed out for proof reading. The class schedules can be printed out at any time in whatever form chosen. With the help of the program the format of the printout is easily specified to conform to users' needs. The program can store many different printing and printer specifications. 
For payroll calculation, a list of all lessons can easily be printed for each teacher. A similar list can also be printed for each class or each room. For daily needs and, for example, for the telephone exchange, tables can be printed which show at a glance the availability of each class, teacher or room.

IIT draws up new schedules every week. Although weeks within one degree program tend to retain the same structure throughout the whole term, the part-time attendance program provide for contact lessons only one week per month; thus we give four different classes in a month and a teacher's timetables for four consecutive weeks may be different. This makes IIT a large-scale consumer of schedules.

After inputting all the lessons, the software prints out individual timetables for

- students (about 500);

- study groups (17 groups in four degree programs);

- teachers (about 70);

- classrooms and computer laboratories (31), and

- course diaries with the names of the attending students.

You can also print information in many condensed forms such as:

- wall tables;

- list of lessons;

- vacant classes;

- non-occupied teachers, and

- vacant class-rooms.

\section{EVALUATION OF THE SOFTWARE}

The Starsoft Primus and Kurre are specially developed for school administrations. The user interface is sophisticated and is based on graphics. Once having fed in the data, i.e., the names of the students, teachers and class-rooms, and the subjects in all curricula, the user works with the mouse in selecting the required data from lists shown by the program. The user hardly needs the keyboard at all and this feature makes errors less likely. It is also more efficient to point to the required data instead of typing it.

The software is very flexible. When first purchased, it contains the most usual registers in a school administration. The user then defines the rest of the contents of all registers and determines in which order he or she works, thus tailoring the software for optimal use in his or her own environment.

The software is available in any version of IBM or compatibles. It runs under MS-DOS, OS or Windows. Primus needs at least an 80286-processor, a 1 Mb RAM, a fast hard disk together with an EGA-screen and a Microsoft-compatible mouse. Primus also works in local networks. Complete transfer of information is possible between Kurre and Primus in both directions.

The producer claims that the software is very flexible and, although I am usually highly sceptical about such claims this one I can endorse. To sum up then: Although I believe that no product is so good that it cannot be made better, StarSoft Primus and Kurre constitute a product I can go a long way toward recommending. One must not forget that planning quality timetables like these is time consuming. Nevertheless, I think that it is time well spent. Awkward, perhaps unsocial, hours on a student's or teacher's schedule will not do much to maintain or improve learning or educational standards. 